\title{
ENTANGLEMENT IN TWO QUBIT MAGNETIC MODELS WITH DM ANTISYMMETRIC ANISOTROPIC EXCHANGE INTERACTION
}

\author{
ZEYNEP NILHAN GURKAN* and OKTAY K. PASHAEV ${ }^{\dagger}$ \\ Department of Mathematics, Izmir Institute of Technology, \\ Urla-Izmir 35430, Turkey \\ *nilhangurkan@iyte.edu.tr \\ †oktaypashaev@iyte.edu.tr
}

Received 15 October 2008

\begin{abstract}
In the present paper, an influence of the anisotropic antisymmetric exchange interaction, the Dzialoshinskii-Moriya (DM) interaction, on entanglement of two qubits in various magnetic spin models, including the pure DM model and the most general $X Y Z$ model, are studied. We find that the time evolution generated by DM interaction can implement the SWAP gate and discuss realistic quasi-one-dimensional magnets where it can be realized. It is shown that inclusion of the DM interaction to any Heisenberg model creates, when it does not exist, or strengthens, when it exists, the entanglement. We give physical explanation of these results by studying the ground state of the systems at $T=0$. Nonanalytic dependence of the concurrence on the DM interaction and its relation with quantum phase transition is indicated. Our results show that spin models with the DM coupling have some potential applications in quantum computations and the DM interaction could be an efficient control parameter of entanglement.
\end{abstract}

Keywords: Entanglement; qubit; spin models; DM interaction; $X Y Z$ model; quantum phase transition; quantum computation; SWAP gate.

\section{Introduction}

The entanglement property has been discussed in the early years of quantum mechanics as a specific quantum mechanical nonlocal correlation ${ }^{1-3}$ and recently, it has become a key point of the quantum information theory. ${ }^{4}$ For entangled subsystems the whole state vector cannot be separated into a product of the subsystem states. This is why these subsystems are no longer independent, even if they are far separated spatially. A measurement on one subsystem not only gives information about the other subsystem, but also provides the possibility of manipulating it. Therefore entanglement becomes the main tool in quantum computations and information processing, quantum cryptography, teleportation, etc. ${ }^{5}$ Due to the intrinsic pairwise character of the entanglement, entangled qubit pairs play a crucial role in such computations. It is clear that single qubit gates are unable to generate entanglement in an $N$ qubit system, and to prepare an entangled state, one 
needs an inter qubit interaction, which is a two qubit gate. The simplest two qubit interaction is described by the Ising one between spin $1 / 2$ particles in the form of $J \sigma_{1}^{z} \sigma_{2}^{z}$. More general interaction between two qubits is given by the Heisenberg magnetic spin interaction models. These models have been extensively studied for several decades, experimentally in condensed matter systems ${ }^{6}$ and theoretically as exactly solvable many body problems (Bethe, Baxter, and others). ${ }^{7,8}$ Now they become promising to realize quantum computation and information processing, by generating entangled qubits and constructing quantum gates ${ }^{9,10}$ in a more general context than the magnetic chains.

Recently, in this way, interaction between two nuclear spins having the Heisenberg form was considered. ${ }^{11}$ The nuclear spins from one side are well isolated from the environment and their decoherence time is sufficiently long. From another side nuclei with spin $1 / 2$ are natural representatives of qubits in quantum information processing, which can realize quantum computational algorithms by using NMR. ${ }^{12-14}$

Very recently entanglement of two qubits ${ }^{15}$ and its dependence on external magnetic fields, anisotropy, and temperature have been considered in several Heisenberg models: the Ising model ${ }^{16-18}$; the $X X$ and $X Y$ models $^{9,19-24}$; the $X X X$ model $^{25}$; the $X X Z$ model $^{26}$; and the $X Y Z$ model. ${ }^{27-29}$ Particularly dependence of entanglement on the type of spin ordering was shown, so that in the isotropic Heisenberg spin chain (the $X X X$ model) spin states are unentangled in the ferromagnetic case $J<0$, while for the antiferromagnetic case $J>0$ entanglement occurs for sufficiently small temperature $T<T_{c}=2 J / k \ln 3$. A significant point in the study of such models is how to increase entanglement in the situation when it already exists or to create entanglement in the situation when it does not exist. Certainly this can be expected from a generalization of bilinear spin-spin interaction of the Heisenberg form. Around 50 years ago, explaining weak ferromagnetism of antiferromagnetic crystals $\left(\alpha-\mathrm{Fe}_{2} \mathrm{O}_{3}, \mathrm{MnCO}_{3}\right.$, and $\left.\mathrm{CrF}_{3}\right)$, has been a controversial problem for a decade, Dzialoshinskii, ${ }^{30}$ from phenomenological arguments, and Moriya ${ }^{31}$ from microscopic grounds, have introduced anisotropic antisymmetric exchange interaction, the Dzialoshinskii-Moriya (DM) interaction, expressed by

$$
\mathbf{D} \cdot\left[\mathbf{S}_{1} \times \mathbf{S}_{2}\right] \text {. }
$$

This interaction arising from extension of the Anderson superexchange interaction theory by including the spin orbit coupling effect, ${ }^{31}$ is important not only for the weak ferromagnetism but also for the spin arrangement in antiferromagnets of low symmetry. In contrast to the Heisenberg interaction which tends to render neighbor spins parallel, the DM interaction has the effect of turning them perpendicular to one another. As we will see in the present paper, it turns out that such spin arrangements are likely to increase entanglement. In most materials with weak ferromagnetism and the DM coupling, parameter $D$ is small compared to $J$. The values reported in the literature range from $(D / J) \approx 0.02$ to 0.07 (see Ref. 40 and references therein). However, in some compounds, the DM interaction can attain a 
sizeable value in comparison with the usual symmetric superexchange $J$. Depending on the compound, its value varies between $(D / J) \approx 0.05$ and 0.2 . Moreover, recently the DM interaction was found to be present in a number of quasi-one-dimensional magnets. ${ }^{41}$ It was found that even the compound $\mathrm{RbCoCl}_{3} \cdot 2 \mathrm{H}_{2} \mathrm{O}$ is described as a pure DM chain. ${ }^{39}$ The low-temperature magnetic behavior of this compound gives strong evidence that the material consists of weakly interacting linear chains with predominant DM interaction. In addition, study of the DM interaction influence on dynamics of the one-dimensional quantum antiferromagnet shows the big difference in the behavior, depending on whether the coupling $D$ is smaller or larger than the exchange interaction $J .{ }^{41}$ All these results imply that a study of spin models with DM interaction could have realistic applications. Then for applications in quantum computations, it poses the problem to find the entanglement dependence on this interaction.

In the present paper, we study the influence of the DM interaction on entanglement of two qubits in all particular magnetic spin models, including the most general $X Y Z$ model. We find that in all cases, inclusion of the DM interaction creates, when it does not exist, or strengthens, when it exists, entanglement. For example, we show that in the case of isotropic Heisenberg $X X X$ model discussed above, inclusion of this term increases entanglement for antiferromagnetic case and for sufficiently strong coupling $D>\left(k T \sinh ^{-1} e^{|J| / k T}-J^{2}\right)^{1 / 2}$ it creates entanglement even in ferromagnetic case. We give detailed physical explanations of these results by studying ground state of the system at $T=0$. In this state, we find nonanalytic dependence of concurrence on the DM interaction and establish its relation with the quantum phase transition. In addition, we show that time evolution generated by DM interaction can be implemented as the SWAP gate. These results indicate that spin models with DM coupling have some potential applications in quantum computations, and DM interaction could be an efficient control parameter of entanglement.

The paper is organized as follows. In Sec. 2, we formulate the general $X Y Z$ model with DM coupling and find the density matrix and eigenvalues for the concurrence. Then we consider the time evolution and its relation with the SWAP gate. Since the concurrence calculation depends on several parameters, in the following sections, we consider all possible particular cases from the unified point of view. We think that such presentation is pedagogical and could be affordable by experimentalists. In Sec. 3, the main properties and entanglement of pure DM model and the relation of this model with SWAP gate are considered. The Ising model with DM interaction is studied in Sec. 4. In particular, realization of the model for description of two nuclear spins with DM coupling and implications for the quantum phase transitions in the presence of magnetic field are given. In Sec. 5, we consider the $X Y$ model and its particular reductions to the $X X$ case, and to the Ising model. We show that inclusion of the transverse magnetic field leads to the different behavior of concurrence $C_{12}$ for the undercritical and the overcritical couplings. For $T=0$, the nonanalytic behavior for $C_{12}(D)$ is found. The $X X X$ 
Heisenberg model is the subject of Sec. 6. Section 7 is devoted to the $X X Z$ model, where the influence of DM coupling and magnetic field on the concurrence and the quantum phase transitions are studied. In Sec. 8, we study $X Y Z$ model in both antiferromagnetic and ferromagnetic cases, with inclusion of the DM coupling. The nonanalytic behavior at $T=0$ is found. In Sec. 9, several implications for future studies are discussed.

\section{2. $X Y Z$ Heisenberg Model}

We start our consideration with the most general $X Y Z$ model, by inclusion of homogeneous $B$ and nonhomogeneous $b$ magnetic fields, and choosing the DM interaction (1) in the form $(\mathbf{D} / 2)=(D / 2) \cdot \mathbf{z}$. Then for two qubits we have Hamiltonian

$$
H=\frac{1}{2}\left[J_{x} \sigma_{1}^{x} \sigma_{2}^{x}+J_{y} \sigma_{1}^{y} \sigma_{2}^{y}+J_{z} \sigma_{1}^{z} \sigma_{2}^{z}+B_{+} \sigma_{1}^{z}+B_{-} \sigma_{2}^{z}+D\left(\sigma_{1}^{x} \sigma_{2}^{y}-\sigma_{1}^{y} \sigma_{2}^{x}\right)\right],
$$

where $B_{+} \equiv B+b, B_{-} \equiv B-b$, and $\sigma_{i}^{x}, \sigma_{i}^{y}, \sigma_{i}^{z}, i=1,2$ denote Pauli matrices related with the first and the second qubits.

\subsection{Eigenvalues and eigenstates}

To study the thermal entanglement in this system, we firstly need to obtain all eigenvalues and eigenstates of the Hamiltonian (2): $H\left|\Psi_{i}\right\rangle=E_{i}\left|\Psi_{i}\right\rangle, i=1,2,3,4$. Simple calculations show that the energy levels are:

$$
E_{1,2}=\frac{J_{z}}{2} \mp \mu, \quad E_{3,4}=-\frac{J_{z}}{2} \mp \nu
$$

where $\mu \equiv \sqrt{B^{2}+J_{-}^{2}}, \nu \equiv \sqrt{b^{2}+J_{+}^{2}+D^{2}}$, and $J_{ \pm} \equiv\left(J_{x} \pm J_{y}\right) / 2$, and the corresponding wave functions are

$$
\left|\Psi_{1,2}\right\rangle=\frac{1}{\sqrt{2 \mu(\mu \pm B)}}\left[\begin{array}{c}
J_{-} \\
0 \\
0 \\
-(B \pm \mu)
\end{array}\right], \quad\left|\Psi_{3,4}\right\rangle=\frac{1}{\sqrt{2 \nu(\nu \mp b)}}\left[\begin{array}{c}
0 \\
(b \mp \nu) \\
J_{+}-i D \\
0
\end{array}\right]
$$

For $B=0, b=0$, and $D=0$, these wave functions reduce to the maximally entangled Bell states

$$
\begin{aligned}
& \left|\Psi_{2,1}\right\rangle \rightarrow\left|B_{0,3}\right\rangle=\frac{1}{\sqrt{2}}(|00\rangle \pm|11\rangle), \\
& \left|\Psi_{4,3}\right\rangle \rightarrow\left|B_{1,2}\right\rangle=\frac{1}{\sqrt{2}}(|01\rangle \pm|10\rangle .
\end{aligned}
$$




\subsection{Time evolution of states and $S W A P$ gate}

Here we like to show the direct relationship between our spin model and quantum gates. For this reason we consider the evolution operator

$$
U(t)=\exp \left[-\frac{i}{\hbar} H t\right]
$$

determined by two qubit Hamiltonian (2) of $X Y Z$ model with DM coupling, $B=0$ and $b=0$. Then evolution of the standard basis is given by

$$
\begin{aligned}
& |00\rangle \rightarrow e^{\frac{-i J_{z} t}{2 \hbar}}\left[\cos \frac{t J_{-}}{\hbar}|00\rangle-i \sin \frac{t J_{-}}{\hbar}|11\rangle\right], \\
& |11\rangle \rightarrow e^{\frac{-i J_{z} t}{2 \hbar}}\left[\cos \frac{t J_{-}}{\hbar}|11\rangle-i \sin \frac{t J_{-}}{\hbar}|00\rangle\right], \\
& |01\rangle \rightarrow e^{\frac{i J_{z} t}{2 \hbar}}\left[\cos \frac{t \nu}{\hbar}|01\rangle-i \frac{J_{+}-i D}{\nu} \sin \frac{t \nu}{\hbar}|10\rangle\right], \\
& |10\rangle \rightarrow e^{\frac{i J_{z} t}{2 \hbar}}\left[\cos \frac{t \nu}{\hbar}|10\rangle-i \frac{J_{+}+i D}{\nu} \sin \frac{t \nu}{\hbar}|01\rangle\right],
\end{aligned}
$$

where $\nu=\sqrt{J_{+}^{2}+D^{2}}$. In particular cases, discussed in the next section, this evolution can implement the SWAP gate at time $t=\hbar \pi / 2 \nu$.

\subsection{Density matrix and concurrence}

State of the system at thermal equilibrium is determined by the density matrix

$$
\rho(T)=\frac{e^{-H / k T}}{\operatorname{Tr}\left[e^{-H / k T}\right]}=\frac{e^{-H / k T}}{Z},
$$

where $Z=\operatorname{Tr}\left[e^{-H / k T}\right]$ is the partition function, $k$ is the Boltzmann constant and $T$ is the temperature. Then by exponentiation of Hamiltonian (2) we find

$$
e^{-H / k T}=\left[\begin{array}{cccc}
A_{11} & 0 & 0 & A_{14} \\
0 & A_{22} & A_{23} & 0 \\
0 & A_{32} & A_{33} & 0 \\
A_{41} & 0 & 0 & A_{44}
\end{array}\right]
$$

where

$$
\begin{aligned}
& A_{11}=e^{\frac{-J_{z}}{2 k T}}\left[\cosh \frac{\mu}{k T}-\frac{B}{\mu} \sinh \frac{\mu}{k T}\right], \\
& A_{44}=e^{-\frac{J_{z}}{2 k T}}\left[\cosh \frac{\mu}{k T}+\frac{B}{\mu} \sinh \frac{\mu}{k T}\right], \\
& A_{14}=-e^{-\frac{J_{z}}{2 k T}} \frac{J_{-}}{\mu} \sinh \frac{\mu}{k T},
\end{aligned}
$$




$$
\begin{aligned}
& A_{41}=-e^{-\frac{J_{z}}{2 k T}} \frac{J_{-}}{\mu} \sinh \frac{\mu}{k T}, \\
& A_{22}=e^{\frac{J_{z}}{2 k T}}\left[\cosh \frac{\nu}{k T}-\frac{b}{\nu} \sinh \frac{\nu}{k T}\right] \text {, } \\
& A_{33}=e^{\frac{J z}{2 k T}}\left[\cosh \frac{\nu}{k T}+\frac{b}{\nu} \sinh \frac{\nu}{k T}\right] \text {, } \\
& A_{23}=-e^{\frac{J_{z}}{2 k T}} \frac{J_{+}+i D}{\nu} \sinh \frac{\nu}{k T} \\
& A_{32}=-e^{\frac{J_{z}}{2 k T}} \frac{J_{+}-i D}{\nu} \sinh \frac{\nu}{k T},
\end{aligned}
$$

and

$$
Z=\operatorname{Tr}\left[e^{-H / k T}\right]=2\left[e^{\frac{-J_{z}}{2 k T}} \cosh \frac{\mu}{k T}+e^{\frac{J z}{2 k T}} \cosh \frac{\nu}{k T}\right]
$$

As $\rho(T)$ represents a thermal state, the entanglement in this state is called the thermal entanglement. The degree of entanglement could be characterized by the concurrence $C_{12}$, which is defined as ${ }^{15,32}$

$$
C_{12}=\max \left\{\lambda_{1}-\lambda_{2}-\lambda_{3}-\lambda_{4}, 0\right\},
$$

where $\lambda_{1} \geq \lambda_{2} \geq \lambda_{3} \geq \lambda_{4}>0$ are the ordered square roots of eigenvalues of the operator

$$
\rho_{12}=\rho\left(\sigma^{y} \otimes \sigma^{y}\right) \rho^{*}\left(\sigma^{y} \otimes \sigma^{y}\right) .
$$

The concurrence is a bounded function $0 \leq C_{12} \leq 1$, so that when $C_{12}=0$, the states are unentangled, while for $C_{12}=1$, the states are maximally entangled.

For the general Hamiltonian (2) we find:

$$
\begin{aligned}
& \lambda_{1,2}=\frac{e^{\frac{-J_{z}}{2 k T}}}{Z}\left|\sqrt{1+\frac{J_{-}^{2}}{\mu^{2}} \sinh ^{2} \frac{\mu}{k T}} \mp \frac{J_{-}}{\mu} \sinh \frac{\mu}{k T}\right|,
\end{aligned}
$$

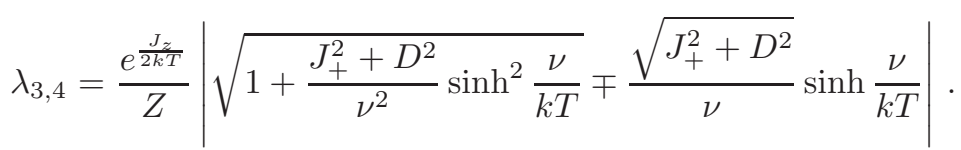

Then, to calculate the concurrence, we need to order these eigenvalues. Since they depend on several parameters, before studying the most general case, it is useful to treat all particular cases separately to clarify the influence of the DM coupling on the entanglement. Starting from pure DM model, we study various Heisenberg models, including the general $X Y Z$ case.

Before this, we just like to stress here the general observation on the concurrence (16). If the biggest eigenvalue say $\lambda_{1}$ is degenerate, then its positive contribution would be compensated by another degenerate one, so that $C_{12}=0$ and states are always unentangled. We will encounter this situation in several cases and it has 
a simple physical explanation. The degenerate biggest eigenvalues of the density matrix correspond to the minimal values of the energy, so that the ground state of the system becomes degenerate and no entanglement occurs.

\section{Pure DM Model}

\subsection{Main characteristics of DM model}

As we discussed in Sec. 1, some realistic quasi-one-dimensional compounds with predominance of DM interaction can be described as a pure DM model. ${ }^{39}$ Here we consider the main characteristic properties of the DM coupling between two qubits and its influence on the entanglement. If in Hamiltonian (2) we put $J_{x}=J_{y}=J_{z}=$ 0 and $B=b=0$ then the model is determined completely by the DM term (1). In this case, the first two eigenstates become degenerate $E_{1}=E_{2}=0$ and $E_{3,4}= \pm D$. For definiteness we choose $D>0$, then for $T=0$ the ground state of the system with energy $E_{4}=-D$ is an entangled state $|10\rangle-i|01\rangle$. When temperature increases, this state becomes mixed with the higher states and entanglement decreases. But for sufficiently large value of $D$, the ground state can be alienated so that entanglement increases. This shows that for a given $D$ there exists $k T_{c}=D / \ln (1+\sqrt{2})$ so that for the under critical case $T<T_{c}$ the states become entangled and the concurrence is $C_{12}=((\sinh (D / k T)-1) /(\cosh (D / k T)+1))$ (see Fig. 1$)$. For $T=0$, the concurrence $C_{12}=1$ and the ground state is maximally entangled.

\subsection{DM model and $S W A P$ gate}

The time evolution in pure DM model from one side is related with the SWAP gate, from another side can create maximally entangled states. In this case according to (8)-(11) for time evolution, we have

$$
U\left(\frac{\pi \hbar}{2 D}\right)|00\rangle=|00\rangle, \quad U\left(\frac{\pi \hbar}{2 D}\right)|11\rangle=|11\rangle
$$

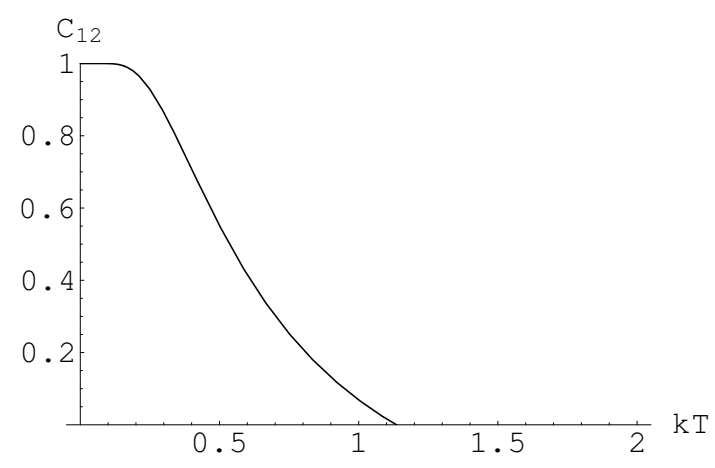

Fig. 1. Concurrence versus temperature for $D=1$ and $T_{c}=1.136$. 


$$
U\left(\frac{\pi \hbar}{2 D}\right)|01\rangle=-|10\rangle, \quad U\left(\frac{\pi \hbar}{2 D}\right)|10\rangle=-|01\rangle .
$$

Therefore, we can see that the operator $U(\pi \hbar / 2 D)$ acts as the SWAP gate. Moreover at time $t=\pi \hbar / 4 D$, the states $|01\rangle$ and $|10\rangle$ become maximally entangled Bell states.

$$
\begin{aligned}
& U\left(\frac{\pi \hbar}{4 D}\right)|01\rangle=\frac{1}{\sqrt{2}}(|01\rangle-|10\rangle), \\
& U\left(\frac{\pi \hbar}{4 D}\right)|10\rangle=\frac{1}{\sqrt{2}}(|10\rangle+|01\rangle) .
\end{aligned}
$$

\section{Ising Model}

For $J_{x}=J_{y}=0, J_{z} \neq 0$ and $B=b=0, D=0$ the Hamiltonian (2) describes the Ising model. It was observed before that for pure Ising model in both antiferromagnetic, $J_{z}>0$, and ferromagnetic cases, $J_{z}<0$, the concurrence is zero and the states are always unentangled. ${ }^{16-18}$ The physical insight of such behavior is easy to understand. When $J_{-}=J_{+}=0$, the density matrix $\rho(12)$ is diagonal in the standard basis, which implies the absence of quantum correlations. Despite having four maximally entangled states as the eigenvectors, the states $\left|\Psi_{1,2}\right\rangle$ and $\left|\Psi_{3,4}\right\rangle$ are degenerated, so that the Ising thermal state has no entanglement. The situation does not change if one includes homogeneous $B$ or nonhomogeneous $b$ magnetic fields, because the density matrix $\rho$ is still diagonal, and no entanglement occurs.

\subsection{Ising model with DM coupling $(B=0, b=0, D \neq 0)$}

In contrast to magnetic fields, which do not create entanglement, inclusion of the DM coupling contributes to the nondiagonal elements of $\rho$ and creates entanglement.

\subsubsection{Antiferromagnetic case $\left(J_{z}>0\right)$}

In this case, addition of the DM coupling to the Ising model splits the degenerate ground state with $E_{3}=E_{4}=-J_{z} / 2$ so that it becomes a singlet with $E_{3}=$ $-\left(\left|J_{z}\right| / 2\right)-D$, for $D>0$ or $E_{4}=-\left(\left|J_{z}\right| / 2\right)+D$, for $D<0$. At $T=0$, this leads to the maximally entangled state with $C_{12}=1$. When temperature increases, the maximally entangled ground state becomes mixed with the higher eigenstates and the entanglement decreases. However, for a given temperature, by increasing the coupling $D>D_{c}$, where $D_{c}=k T \sinh ^{-1} e^{-J_{z} / k T}$, we can decrease this mixture and increase entanglement, so that the concurrence is

$$
C_{12}=\frac{\sinh \frac{|D|}{k T}-e^{-J_{z} / k T}}{\cosh \frac{|D|}{k T}+e^{-J_{z} / k T}} .
$$




\subsubsection{Ferromagnetic case $\left(J_{z}<0\right)$}

In this case, the ground state for small $D$ at $T=0$ is also a doublet and no entanglement occurs. However, with growing $D$, the eigenstate $E_{3}=\left(\left|J_{z}\right| / 2\right)-D$ is lowering so that at critical value $D_{c}=\left|J_{z}\right|$, the ground state becomes triplet. When $D>D_{c}$, the ground state $E_{3}$ is maximally entangled singlet. With growing temperature, a mixture of this state with the higher states decreases entanglement. For given temperature $T$, there exist the critical value $D_{c}=\left|J_{z}\right|+(k T / 2) \ln (1+$ $\left.e^{-2\left|J_{z}\right| / k T}\right)$ so that for $D>D_{c}$ the concurrence is

$$
C_{12}=\frac{\sinh \frac{|D|}{k T}-e^{\left|J_{z}\right| / k T}}{\cosh \frac{|D|}{k T}+e^{\left|J_{z}\right| / k T}} .
$$

Comparison of (23) and (24) shows that in the antiferromagnetic case, the states can be entangled more easily than in the ferromagnetic one.

\subsection{Ising model for two nuclear spins with DM coupling}

As an application of the above calculations, here we discuss the entanglement of two nuclear spins. Recently, two nuclear spins were considered in a model with weak Heisenberg type interaction in a constant longitudinal magnetic field along $z$ direction $^{11}$

$$
\begin{aligned}
H & =H_{z}+H_{x y}, \\
H_{z} & =-\frac{1}{2}\left(\omega_{1} \sigma_{1}^{z}+\omega_{2} \sigma_{2}^{z}+J \sigma_{1}^{z} \sigma_{2}^{z}\right), \\
H_{x y} & =-\frac{1}{2}\left(J \sigma_{1}^{x} \sigma_{2}^{x}+J \sigma_{1}^{y} \sigma_{2}^{y}\right),
\end{aligned}
$$

where the isotropic form for the spin coupling $J$ is assumed, and $\omega_{1,2} \equiv(B \mp b)$ are the Larmor frequencies of two nuclear spins, $\hbar=1$. In the experiments, two different nuclear spins are selected, $\omega_{1} \neq \omega_{2}$ (we assume $\omega_{1}>\omega_{2}$ ), and the longitudinal constant magnetic field is in the order of $1 \mathrm{THz}$, so that $\omega_{1}$ and $\omega_{2}$ are much larger than $J$ and $\eta=J /\left(\omega_{1}-\omega_{2}\right) \ll 1$. $H_{x y}$ is nondiagonal in $\sigma_{z}$ representation and due to quantum fluctuations of order $\eta^{2}$, it can be ignored. Thus, the Ising part $H_{z}$ of the Hamiltonian is a well precise approximation. ${ }^{11}$ However, as we have seen above, for the Ising model with external magnetic fields no entanglement occurs, this is why two nuclear spins in this model are unentangled for any $\omega_{1}$ and $\omega_{2}$. From another viewpoint, as follows from our consideration in Sec. 4.1, the addition of an interaction between qubits in the form of the DM coupling could make them entangled. Now by adding the DM interaction to two nuclear spin Hamiltonian (26) we get the Ising model with homogeneous magnetic field $B$, nonhomogeneous magnetic field $b$, and the DM interaction $D$. In the antiferromagnetic and the ferromagnetic cases, when $J_{z}= \pm\left|J_{z}\right|$, respectively, for sufficiently strong $D>D_{c}$, 
where $\left(D_{c} / \sqrt{D_{c}^{2}+b^{2}}\right) \sinh \left(\sqrt{D_{c}^{2}+b^{2}} / k T\right)=e^{\mp \frac{\left|J_{z}\right|}{k T}}$, the states become entangled and the concurrence is

$$
C_{12}=\frac{\frac{D}{\nu} \sinh \frac{\nu}{k T}-e^{\mp \frac{\left|J_{z}\right|}{k T}}}{\cosh \frac{\nu}{k T}+\cosh \frac{B}{k T} e^{\mp \frac{\left|J_{z}\right|}{k T}}},
$$

where $B=\left(\omega_{1}+\omega_{2}\right) / 2, b=\left(\omega_{1}-\omega_{2}\right) / 2$, and $\nu=\sqrt{\left(\left(\omega_{2}-\omega_{1}\right)^{2} / 4\right)+D^{2}}$. It is worth to note that the homogeneous magnetic field $B$ does not change the critical value for the entanglement, but could change the level of the entanglement. Moreover, increasing magnetic field decreases value of the entanglement. It turns out that for the system at $T=0$, the concurrence becomes nonanalytic when $D=D_{c}$

$$
C_{12}= \begin{cases}\frac{D}{\nu}, & \nu>B \mp\left|J_{z}\right| ; \\ \frac{D}{2 \nu}, & \nu=B \mp\left|J_{z}\right| ; \\ 0, & \nu<B \mp\left|J_{z}\right|,\end{cases}
$$

which implies quantum phase transitions at the critical value $D_{c}=\left(B \mp\left|J_{z}\right|\right)^{2}-b^{2}$.

\subsection{Ising model with DM coupling and $S W A P$ gate}

If $J_{x}=J_{y}=0$ but $J_{z}$ and $D$ are nonvanishing and related by $J_{z}=8 n D,(n=$ $\pm 1, \pm 2, \ldots)$, then again like in Sec. 3.2 the evolution operator $U(\pi \hbar / 2 D)$ acts as the SWAP gate. Our consideration shows that the Ising model, which was derived in several physical situations for interaction of qubits, with addition of the DM coupling, from one side leads to entanglement of states, from another side it can model the SWAP gate as in (19) and (20). This result shows that the Ising model with DM coupling has some potential applications in quantum computations.

\section{5. $X Y$ Heisenberg Model}

In the pure $X Y$ Heisenberg model $J_{z}=0, J_{x} \neq J_{y}$ and $B=0, b=0, D=0$ in (2), for the antiferromagnetic case $J_{x}>0, J_{y}>0$ the ordered eigenvalues are $\lambda_{3}>\lambda_{1}>\lambda_{2}>\lambda_{4}$ and for $\sinh \left(J_{+} / k T\right)>\cosh \left(J_{-} / k T\right)$ the entanglement occurs with $C_{12}=\left(\sinh \left(J_{+} / k T\right)-\cosh \left(J_{-} / k T\right)\right) /\left(\cosh \left(J_{-} / k T\right)+\cosh \left(J_{+} / k T\right)\right)$. In the ferromagnetic case $J_{x}<0, J_{y}<0$, the entanglement occurs when $\sinh \left(\left|J_{-}\right| / k T\right)>$ $\cosh \left(J_{+} / k T\right)$ with the concurrence $20,21,23,24$

$$
C_{12}=\frac{\sinh \frac{\left|J_{+}\right|}{k T}-\cosh \frac{J_{-}}{k T}}{\cosh \frac{\left|J_{-}\right|}{k T}+\cosh \frac{J_{+}}{k T}} .
$$


For the particular case of pure $X X$ model, when $J_{x}=J_{y} \equiv J$, in both antiferromagnetic and ferromagnetic cases, the states become entangled at sufficiently small temperature

$$
T<T_{c}=\frac{|J|}{k \sinh ^{-1} 1} .
$$

As was shown in Refs. 9, 10, 19 and 27, inclusion of the magnetic field does not change this critical temperature.

\section{1. $X Y$ Heisenberg model with DM coupling $(B=0, b=0$, $D \neq 0$ )}

By addition of the DM coupling, eigenvalues become $\lambda_{1,2}=\left(e^{ \pm J_{-} / k T}\right) / Z, \lambda_{3,4}=$ $\left(e^{ \pm \sqrt{J_{+}^{2}+D^{2}} / k T}\right) / Z$ where $Z=2\left[\cosh \left(\left|J_{-}\right| / k T\right)+\cosh \left(\sqrt{J_{+}^{2}+D^{2}}\right) / k T\right]$.

In the antiferromagnetic case for any temperature $T$ we can adjust sufficiently strong DM coupling $D$ so that for $\sinh \left(\sqrt{J_{+}^{2}+D^{2}} / k T\right)>\cosh \left(J_{-} / k T\right)$ the entanglement occurs with concurrence

$$
C_{12}=\frac{\sinh \frac{\sqrt{J_{+}^{2}+D^{2}}}{k T}-\cosh \frac{J_{-}}{k T}}{\cosh \frac{\sqrt{J_{+}^{2}+D^{2}}}{k T}+\cosh \frac{J_{-}}{k T}} .
$$

The ferromagnetic case gives the same result as the antiferromagnetic one. The comparison with pure $X Y$ model shows that the level of entanglement increases with growing coupling $D$.

In the particular case $J_{x}=J_{y} \equiv J$, the ordered eigenvalues are $\lambda_{4}=$ $\left(e^{\nu / k T} / Z\right)>\lambda_{3}=\left(e^{-\nu / k T} / Z\right)>\lambda_{1,2}=1 / Z$, where $\nu=\sqrt{J^{2}+D^{2}}$ and $Z=2(1+\cosh (\nu / k T))$. Then the entanglement occurs when $\sinh (\nu / k T)>1$ and the concurrence is $C_{12}=(\sinh (\nu / k T)-1) /(\cosh (\nu / k T)+1)$. Comparison with the pure $X X$ model (31) shows that the critical temperature,

$$
T_{c}=\frac{\sqrt{J^{2}+D^{2}}}{k \sinh ^{-1} 1},
$$

in this case increases with growing $D$. For $D=0\left|\Psi_{3}\right\rangle$ in (4) is the ground state with eigenvalue $E_{3}=-\left|J_{+}\right|$, which is the maximally entangled Bell state, so that the concurrence $C_{12}=1$. As $T$ increases the concurrence decreases due to the mixing of other states with this maximally entangled one. ${ }^{\mathrm{a}}$

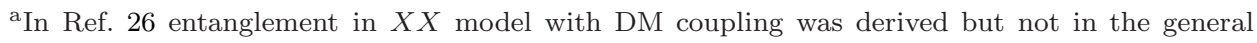
$X X Z$ case as it is claimed in the paper. 


\subsection{Ising model in transverse magnetic field}

As a particular case of the general $X Y$ model now we consider the transverse Ising model, when $J_{y}=0$, with external magnetic field $B$ in $z$-direction, ${ }^{21}$ and with addition of DM interaction:

$$
H=\frac{1}{2}\left[J_{x}\left(\sigma_{1}^{x} \sigma_{2}^{x}\right)+B\left(\sigma_{1}^{z}+\sigma_{2}^{z}\right)+D\left(\sigma_{1}^{x} \sigma_{2}^{y}-\sigma_{1}^{y} \sigma_{2}^{x}\right)\right] .
$$

The corresponding eigenvalues and the partition function $Z$ can be written as follows

$$
\begin{aligned}
& \lambda_{1,2}=\frac{1}{Z}\left|\sqrt{1+\frac{J^{2}}{B^{2}+J^{2}} \sinh ^{2} \frac{\sqrt{B^{2}+J^{2}}}{k T}} \mp \frac{J}{\sqrt{B^{2}+J^{2}}} \sinh \frac{\sqrt{B^{2}+J^{2}}}{k T}\right|, \\
& \lambda_{3,4}=\frac{1}{Z} e^{\mp \frac{\sqrt{J^{2}+D^{2}}}{k T}}, \\
& Z=2\left[\cosh \frac{\sqrt{B^{2}+J^{2}}}{k T}+\cosh \frac{\sqrt{D^{2}+J^{2}}}{k T}\right] .
\end{aligned}
$$

To find the maximal eigenvalue we compare the difference of $\lambda_{4}$ and $\lambda_{2}$ as a function of $B, D$, and $T, \lambda_{4}-\lambda_{2} \equiv f(B, D, T)$ :

$$
f=e^{\frac{\sqrt{J^{2}+D^{2}}}{k T}}-\sqrt{1+\frac{J^{2}}{B^{2}+J^{2}} \sinh ^{2} \frac{\sqrt{B^{2}+J^{2}}}{k T}}-\frac{J}{\sqrt{B^{2}+J^{2}}} \sinh \frac{\sqrt{B^{2}+J^{2}}}{k T} .
$$

When $f(B, D, T)=0$ we find the critical $D=D_{c}(B, T)$ as

$D_{c}(B, T)$

$$
=\sqrt{-J^{2}+T^{2}\left(\ln \left[\sqrt{1+\frac{J^{2}}{B^{2}+J^{2}} \sinh ^{2} \frac{\sqrt{B^{2}+J^{2}}}{k T}}+\frac{J}{\sqrt{B^{2}+J^{2}}} \sinh \frac{\sqrt{B^{2}+J^{2}}}{k T}\right]\right)^{2}} .
$$

In Fig. 2, we plot $D_{c}$ as a function of $T$ for different values of magnetic field $B=0.05,0.5,0.7,1(J=1, k=1)$. The $3 \mathrm{D}$ plot of $D_{c}$ as a function of $B$ and $T$ for the same values of parameters is given in Fig. 3 .

For critical $D=D_{c}$, the eigenvalues are degenerate $\lambda_{2}=\lambda_{4}$ and as a result the concurrence $C_{12}\left(B, D_{c}, T\right)=0$. However the value of concurrence is different for the under-critical and the over-critical cases. In under-critical case when $D<D_{c}$ the maximal eigenvalue is $\lambda_{2}$ and for the concurrence we have

$$
C_{12}=\max \left\{\frac{\frac{J}{\sqrt{B^{2}+J^{2}}} \sinh \frac{\sqrt{B^{2}+J^{2}}}{k T}-\cosh \frac{\sqrt{D^{2}+J^{2}}}{k T}}{\cosh \frac{\sqrt{B^{2}+J^{2}}}{k T}+\cosh \frac{\sqrt{D^{2}+J^{2}}}{k T}}, 0\right\},
$$



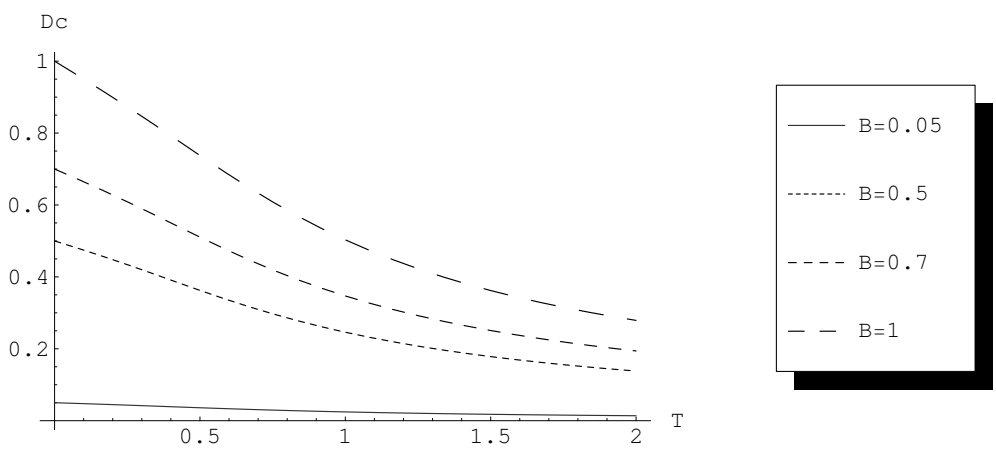

Fig. 2. $D_{c}$ versus $T$ for $B=0.05,0.5,0.7,1$.

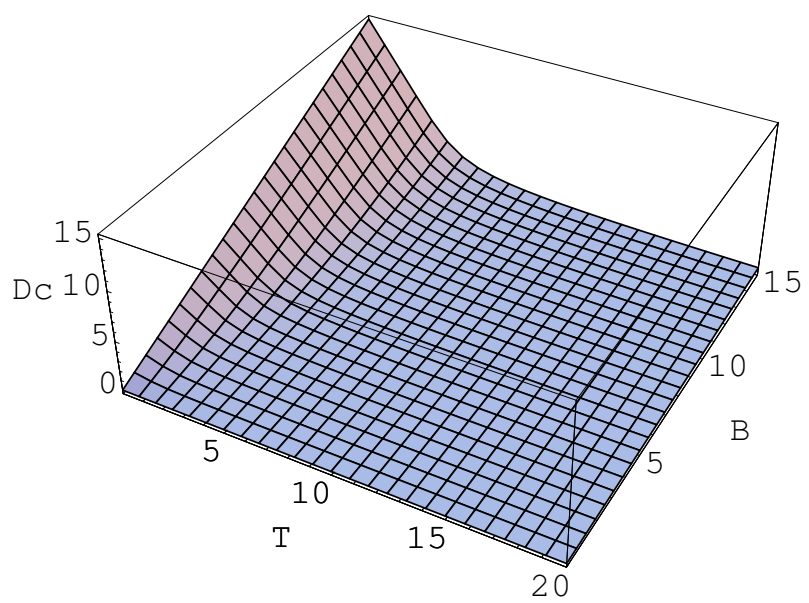

Fig. 3. 3D plot $D_{c}$ versus $B$ and $T$.

while in the over-critical case, when $D>D_{c}$, the maximum eigenvalue is $\lambda_{4}$ and the concurrence is

$$
C_{12}=\max \left\{\frac{\sinh \frac{\sqrt{D^{2}+J^{2}}}{k T}-\sqrt{1+\frac{J^{2}}{B^{2}+J^{2}} \sinh ^{2} \frac{\sqrt{B^{2}+J^{2}}}{k T}}}{\cosh \frac{\sqrt{B^{2}+J^{2}}}{k T}+\cosh \frac{\sqrt{D^{2}+J^{2}}}{k T}}, 0\right\} .
$$

In pure Ising model when $B=0$ and $D=0$ as we can see from (38) we have $f(0,0, T)=0$ and no entanglement occurs. But as reported in Ref. 21, an addition of the transverse magnetic field to the Ising model could create entanglement. Now we can generalize these results by analyzing in addition the influence of DM interaction on entanglement in the Ising model with the magnetic field. When $B=0$, the addition of solely DM term creates entanglement at sufficiently strong $D$, and this value of $D$ becomes bigger for higher temperatures. If we have both terms $B \neq 0$ and 

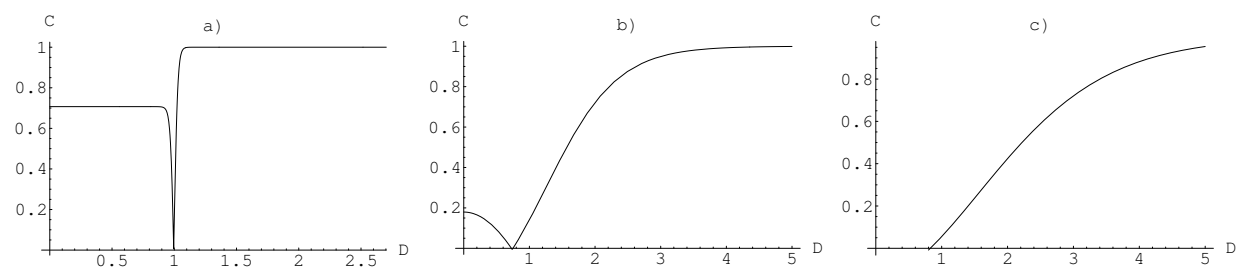

Fig. 4. Concurrence of Ising model in transverse magnetic field versus $D$, when $B=1$ and $T=0.01,0.5,1$.

$D \neq 0$, then with increasing $D$, the behavior of entanglement becomes nontrivial. In Figs. 4(a)-4(c), we show the behavior of entanglement as a function of $D$ for different temperatures. When $T=0$, entanglement is a nonanalytic function of $D$, given by the step function

$$
C_{12}(D)= \begin{cases}\frac{J}{\sqrt{J^{2}+B^{2}}}, & D<D_{c} \\ 0, & D=D_{c} \\ 1, & D>D_{c}\end{cases}
$$

where $D_{c}=B$ [see Fig. 4(a)]. This nonanalytic behavior signals on the quantum phase transition ${ }^{33}$ appearing at $D=D_{c}=1$. In Fig. 4 (b) at temperature $T=0.5$ the entanglement as a function of $D$ decreases down to zero and at $D_{c} \approx 0.75$ reaches its nondifferentiable minima. After this, it increases monotonically with growing $D$. For higher temperature $T=1$ as shown in Fig. 4(c), the entanglement is zero until $D$ becomes sufficiently strong at $D=D_{c}$, where entanglement appears and monotonically grows with growing $D$.

\section{6. $X X X$ Heisenberg Model}

In pure $X X X$ model $J_{x}=J_{y}=J_{z} \equiv J$ and $B=b=D=0$ in (2), entanglement behavior for the ferromagnetic and the antiferromagnetic cases is different. In the spectrum of the model we have three degenerate eigenstates with eigenvalue $J / 2$ and one eigenstate with eigenvalue $-3 J / 2$. It was observed before ${ }^{25}$ that for the ferromagnetic case $(J<0)$ the concurrence is zero and the states are always unentangled. It happens because when $J<0$, the ground state of the system is an equal mixture of the triplet states with energy, $E_{1}=E_{2}=E_{4}=-(|J| / 2)$. The density matrix $\rho$ is diagonal and inclusion of magnetic field does not change the result. Increasing temperature $T$ just increases the singlet mixture with the triplet, which can only decrease entanglement. ${ }^{25,34}$ The situation is different for the antiferromagnetic case when $J>0$. In this case, the ground state is the maximally entangled singlet state with $E_{3}=-(3 J / 2)$, so that the concurrence $C_{12}=1$ at $T=0$. It decreases with $T$ due to mixing of the triplet higher states with the singlet ground state. For a given coupling constant $J$ entanglement occurs at temperature $T<(2 J / k \ln 3) .{ }^{26}$ 


\section{1. $X X X$ Heisenberg model with DM coupling $(B=0, b=0$, $D \neq 0$ )}

Now by adding DM coupling for the antiferromagnetic and the ferromagnetic cases, for $J= \pm|J|$, respectively, for a given temperature $T$ the entanglement occurs when $D>D_{c}=\sqrt{\left(k T \sinh ^{-1} e^{\mp|J| / k T}\right)^{2}-J^{2}}$ with the concurrence

$$
C_{12}=\frac{\sinh \frac{\sqrt{J^{2}+D^{2}}}{k T}-e^{\mp|J| / k T}}{e^{\mp|J| / k T}+\cosh \frac{\sqrt{J^{2}+D^{2}}}{k T}} .
$$

As we can see, inclusion of the DM coupling, in the $X X X$ model, increases entanglement in the antiferromagnetic case and creates entanglement even in the ferromagnetic case. This can be explained if we consider the eigenvalues of our Hamiltonian varying with $D$.

For the antiferromagnetic case, the ground state of the system remains singlet with energy $E_{3}=-(|J| / 2)-\sqrt{J^{2}+D^{2}}$, while from degenerate excited triplet state one of the energy levels $E_{4}=-(|J| / 2)+\sqrt{J^{2}+D^{2}}$ is splitting up. With increasing coupling $D$ the gap between ground state and the first excited doublet state is increasing, this is why the system becomes more entangled.

In the ferromagnetic case, from unentangled triplet ground state one of the states splits with the energy $E_{3}=(|J| / 2)-\sqrt{J^{2}+D^{2}}$. Then at temperature zero this state becomes maximally entangled ground state. This way the DM interaction creates entanglement in the ferromagnetic case. With increasing $D$ the gap between singlet ground state and the first doublet state increases, this is why entanglement in the ferromagnetic case increases.

\section{7. $X X Z$ Heisenberg Model}

When $J_{x}=J_{y}=J \neq J_{z}$ the Hamiltonian (2) becomes

$$
H=\frac{1}{2}\left[J\left(\sigma_{1}^{x} \sigma_{2}^{x}+\sigma_{1}^{y} \sigma_{2}^{y}+\Delta \sigma_{1}^{z} \sigma_{2}^{z}\right)+B_{+} \sigma_{1}^{z}+B_{-} \sigma_{2}^{z}+D\left(\sigma_{1}^{x} \sigma_{2}^{y}-\sigma_{1}^{y} \sigma_{2}^{x}\right)\right],
$$

where $\Delta \equiv\left(J_{z} / J\right)$.

- In a pure $X X Z$ ferromagnetic model, when $J_{z}<0$ and $-\left|J_{z}\right|<J<\left|J_{z}\right|$ or $|\Delta|>1$, we have the degenerate maximal eigenvalues $\lambda_{1}=\lambda_{2}$ and no entanglement occurs. This happens since the ground state of the system is doublet with eigenvalues $E_{1}=E_{2}=-\left(\left|J_{z}\right| / 2\right)$.

- In particular case $|\Delta|=1$ or $|J|=\left|J_{z}\right|$ we have reduction to the $X X X$ model, where the energy level $E_{3}$ merges to the ground state, and the last one becomes triplet state, as we discussed above in Sec. 6 . 
- For $J>0$ and $\Delta>-1$ the maximal eigenvalue is $\lambda_{3}$ and the states are entangled when $\sinh (J / k T)>e^{-J_{z} / k T}$ with the concurrence

$$
C_{12}=\frac{\sinh \frac{J}{k T}-e^{-J_{z} / k T}}{\cosh \frac{J}{k T}+e^{-J_{z} / k T}} .
$$

- For $J<0$ and $\Delta<1$ the maximal eigenvalue is $\lambda_{4}$ and the states are entangled for $\sinh (|J| / k T)>e^{-J_{z} / k T}$ with the concurrence

$$
C_{12}=\frac{\sinh \frac{|J|}{k T}-e^{-J_{z} / k T}}{\cosh \frac{|J|}{k T}+e^{-J_{z} / k T}}
$$

\section{1. $X X Z$ Heisenberg model with $D M$ coupling $(B=0, b=0$,} $D \neq 0$ )

With addition of the DM coupling we have the eigenvalues

$$
\lambda_{1,2}=\frac{1}{2\left[1+e^{J_{z} / k T} \cosh \frac{\sqrt{J^{2}+D^{2}}}{k T}\right]}, \quad \lambda_{3,4}=\frac{e^{\mp \sqrt{J^{2}+D^{2}} / k T}}{2\left[e^{-J_{z} / k T}+\cosh \frac{\sqrt{J^{2}+D^{2}}}{k T}\right]} .
$$

Then for $J_{z}<0$ and $\left|J_{z}\right|>|J|$, there exists critical value $D_{c}=\sqrt{J_{z}^{2}-J^{2}}$ so that for $D>D_{c}$ and $\sinh \left(\sqrt{J^{2}+D^{2}} / k T\right)>e^{-J_{z} / k T}$ the states are entangled with the concurrence

$$
C_{12}=\frac{\sinh \frac{\sqrt{J^{2}+D^{2}}}{k T}-e^{\left|J_{z}\right| / k T}}{\cosh \frac{\sqrt{J^{2}+D^{2}}}{k T}+e^{\left|J_{z}\right| / k T}}
$$

This happens because for $J_{z}<0,\left|J_{z}\right|>|J|$ and $D=0$, the ground state is doublet with $E_{1}=E_{2}=-\left(\left|J_{z}\right| / 2\right)$, and by increasing $D$ so that $D>D_{c}$, the higher energy level $E_{3}$ lowers to the singlet ground state which is maximally entangled. Comparison of (48) with (46) shows that with growing $D$ entanglement increases.

It is worth to note that the concurrence (48) for both signs of $J$ is the same. Moreover, as easy to see in (48) parameters $J$ and $D$ appear symmetrically. It means that the concurrence could be increased by growing $J$ with fixed $D$ either by growing $D$ with fixed $J$. This reflects the known result ${ }^{35}$ on equivalence of the Heisenberg $X X Z$ model with DM coupling to pure $X X Z$ model with modified anisotropy parameter and a certain type of boundary conditions. In fact comparing entanglement in our formulas for pure antiferromagnetic case (46) with the one including the DM interaction (48), we can see that the concurrences are connected by the replacement $J \rightarrow J \sqrt{1+\left(D^{2} / J^{2}\right)}$, which corresponds to the substitution for the anisotropy parameter in the pure $X X Z$ model as $\Delta \rightarrow\left(\Delta / \sqrt{1+\left(\Delta^{2} D^{2}\right) / J_{z}^{2}}\right)$. 


\section{2. $X X Z$ Heisenberg model with DM coupling and magnetic field}

If we take into account the DM interaction $D$ and magnetic field $B$ simultaneously, the above results for critical value of the DM coupling are still valid, but the level of entanglement decreases according to

$$
C_{12}=\frac{\sinh \frac{\sqrt{J^{2}+D^{2}}}{k T}-e^{-J_{z} / k T}}{\cosh \frac{\sqrt{J^{2}+D^{2}}}{k T}+e^{-J_{z} / k T} \cosh \frac{B}{k T}} .
$$

For $T=0$ and $J_{z}>0$ we have nonanalytic behavior

$$
C_{12}= \begin{cases}1, & \sqrt{D^{2}+J^{2}}>B-J_{z} \\ \frac{1}{2}, & \sqrt{D^{2}+J^{2}}=B-J_{z} \\ 0, & \sqrt{D^{2}+J^{2}}<B-J_{z}\end{cases}
$$

which signals appearance of quantum phase transitions. The concurrence versus temperature for different values of coupling $D$ is shown in Fig. 5 , where $J=1, J_{z}=$ 0.5 and magnetic field $B=2$. As we can see in general the entanglement decreases with growing temperature. However, we like to emphasize that for $D<D_{c}$ in Fig. 5(a), when $D=0.1$, the entanglement is increasing with growing temperature. This phenomena can be explained by the fact that for such values of the parameters at $T=0$ the ground state is the separable state with energy $E_{1}=\left(J_{z} / 2\right)-B=$ -1.75 , and the concurrence is zero (see the last case in Eq. (50)). When temperature increases the entangled state with energy $E_{3}=\left(-J_{z} / 2\right) \mp \sqrt{J^{2}+D^{2}}=-1.255$ becomes involved into the mixture and entanglement is increasing.

When $D=D_{c}$ the entanglement decreases smoothly from $C_{12}=0.5$ (Fig. 5(b), $\left.D_{c}=1.118\right)$. By increasing $D(D=1.19)$, first it gives sharp decrease from $C_{12}=1$ [Fig. 5(c)] and then it vanishes slowly. When $D$ becomes bigger $(D=3)$ entanglement decreases slowly from $C_{12}=1$ [Fig. 5(d)].

We compare the concurrence versus magnetic field for different temperatures, when $D=0$ (Fig. 6) and when $D=2$ (Fig. 7). In both cases at $T=0$ the entanglement vanishes abruptly as $B$ crosses critical value $B_{c}=\sqrt{B^{2}+J^{2}}+J_{z}$. This special point $T=0, B=B_{c}$ at which entanglement becomes nonanalytic function of $B$, is the point of quantum phase transition. Comparison of Figs. 6 and 7 shows that the critical value of $B$ at which entanglement disappears suddenly is
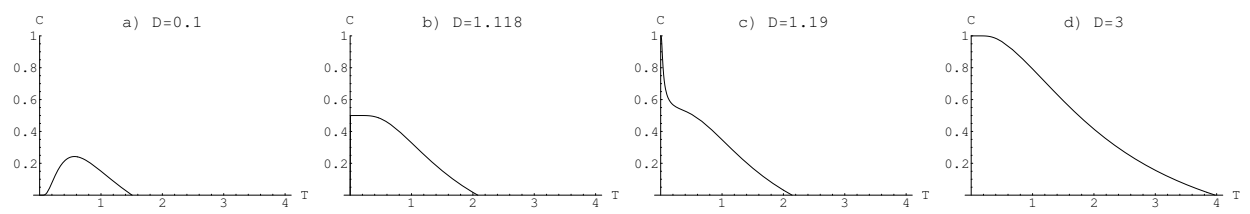

Fig. 5. Concurrence in $X X Z$ model versus temperature for $B=2$ and (a) $D=0.1$, (b) $D=$ 1.118 , (c) $D=1.19$, and (d) $D=3$. 

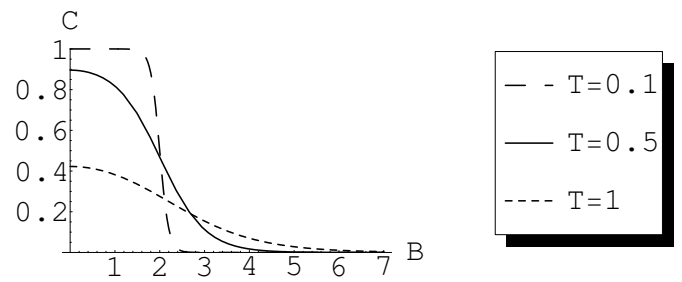

Fig. 6. Concurrence versus magnetic field $B$ for $D=0$ and $T=0.1,0.5,1$.
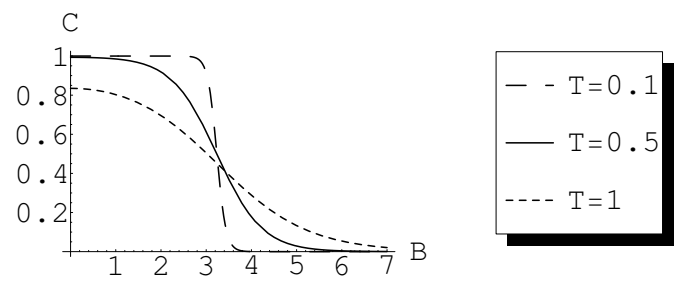

Fig. 7. Concurrence versus magnetic field $B$ for $D=2$ and $T=0.1,0.5,1$.

growing with increasing coupling $D$ : in Fig. $6, B_{c}=2$ and in Fig. $7, B_{c}=3.3$. It shows again that increasing DM coupling improves entanglement.

\section{8. $X Y Z$ Heisenberg Model}

In the present paper, we are not going to analyze all possibilities for the $X Y Z$ model. Instead we restrict ourselves with a particular range of parameters to study the influence of DM coupling in detail.

\subsection{Antiferromagnetic case}

\subsubsection{Pure antiferromagnetic model}

We start from the pure $X Y Z$ model, where for determinacy we chose $J_{z}>J_{y}>$ $J_{x}>0$ implying $J_{+}>\left|J_{-}\right|>0, J_{-}=-\left|J_{-}\right|<0$. Eigenstates of the Hamiltonian (2) are $E_{1,2}=\left(\left|J_{z}\right| / 2\right) \pm\left|J_{-}\right|$and $E_{3,4}=-\left(\left|J_{z}\right| / 2\right) \mp\left|J_{+}\right|$. For zero temperature the ground state is maximally entangled Bell state $|01\rangle-|10\rangle$ with the energy $E_{3}=-\left(\left|J_{z}\right| / 2\right)-\left|J_{+}\right|$. When temperature increases, the state mixes with higher states decreasing entanglement. To find concurrence we have the biggest eigenvalue $\lambda_{4}=(1 / Z) \exp \left(\left|J_{z}\right|+2\left|J_{+}\right|\right) / 2 k T$ and

$$
C_{12}=\max \left\{\frac{\sinh \frac{J_{+}}{k T}-\cosh \frac{J_{-}}{k T} e^{-J_{z} / k T}}{\cosh \frac{J_{+}}{k T}+\cosh \frac{J_{-}}{k T} e^{-J_{z} / k T}}, 0\right\} .
$$

Then entanglement occurs when $\sinh \left(J_{+} / k T\right)>\cosh \left(J_{-} / k T\right) e^{-J_{z} / k T}$. It shows that entanglement depends essentially on the anisotropy, and grows with $J_{+}$and decreases with $J_{-} \cdot{ }^{28}$ 


\subsubsection{XYZ model with DM coupling}

Inclusion of the DM coupling, remains the energy levels $E_{1}$ and $E_{2}$ the same as above, while $E_{3,4}=-\left(\left|J_{z}\right| / 2\right) \mp \sqrt{J_{+}^{2}+D^{2}}$. In this case, the ground state continues to be entangled state but with the energy $E_{3}$. With growing temperature, mixing of this state with the higher states decreases the entanglement. If we consider the difference between two lower states $E_{4}-E_{3}=\sqrt{J_{+}^{2}+D^{2}}$, then by increasing the coupling $D$, it can be made arbitrarily large, so that the entanglement will increase. For $D \gg\left|J_{+}\right|$the state would be maximally entangled.

At $T=0$ the concurrence

$$
C_{12}= \begin{cases}1, & \sqrt{D^{2}+J_{+}^{2}}>J_{-}-J_{z} ; \\ 0, & \sqrt{D^{2}+J_{+}^{2}}=J_{-}-J_{z} ; \\ 1, & \sqrt{D^{2}+J_{+}^{2}}<J_{-}-J_{z},\end{cases}
$$

is nonanalytic function in $D$, and it signals about the quantum phase transition at $D=D_{c}$ where $\sqrt{D_{c}^{2}+J_{+}^{2}}=J_{-}-J_{z}$. When the temperature increases, entanglement occurs for

$$
\sinh \frac{\sqrt{J_{+}^{2}+D^{2}}}{k T}>e^{-J_{z} / k T} \cosh \frac{J_{-}}{k T},
$$

and the concurrence

$$
C_{12}=\frac{\sinh \frac{\nu}{k T}-e^{-J_{z} / k T} \cosh \frac{J_{-}}{k T}}{\cosh \frac{\nu}{k T}+e^{-J_{z} / k T} \cosh \frac{J_{-}}{k T}},
$$

increases with growing anisotropy $J_{+}$and the coupling $D$.

\subsection{Ferromagnetic case $\left(J_{z}<J_{y}<J_{x}<0\right)$}

\subsubsection{Pure $X Y Z$ model}

Let $J_{z}<J_{y}<J_{x}<0$ then $J_{+}=-\left|J_{+}\right|, J_{-}=\left|J_{-}\right|>0$ and $J_{z}=-\left|J_{z}\right|$. For pure $X Y Z$ model, eigenstates of the Hamiltonian are $E_{1,2}=-\left(\left|J_{z}\right| / 2\right) \mp\left|J_{-}\right|$and $E_{3,4}=$ $\left(\left|J_{z}\right| / 2\right) \pm\left|J_{+}\right|$. For zero temperature, the ground state is maximally entangled Bell state $|00\rangle-|11\rangle$ with the energy $E_{1}=-\left(\left|J_{z}\right| / 2\right)-\left|J_{-}\right|$. With increasing temperature, this state mixes with other states and entanglement decreases so that the concurrence is

$$
C_{12}=\frac{\sinh \frac{\left|J_{-}\right|}{k T}-\cosh \frac{\left|J_{+}\right|}{k T} e^{-\left|J_{z}\right| / k T}}{\cosh \frac{\left|J_{-}\right|}{k T}+\cosh \frac{\left|J_{+}\right|}{k T} e^{-\left|J_{z}\right| / k T}}
$$


When temperature reaches the critical value $T=T_{c}$, given by a solution of the following transcendental equation

$$
\sinh \frac{\left|J_{-}\right|}{k T_{c}}=\cosh \frac{\left|J_{+}\right|}{k T_{c}} e^{-\left|J_{z}\right| / k T},
$$

the concurrence vanishes and state becomes unentangled.

\subsection{2. $X Y Z$ model with DM coupling}

With inclusion of the DM coupling, the first couple of energy levels is the same $E_{1,2}=\left(\left(-\left|J_{z}\right|\right) / 2\right) \mp\left|J_{-}\right|$while the second couple becomes $E_{3,4}=\left(\left(\left|J_{z}\right|\right) / 2\right) \mp$ $\sqrt{J_{+}^{2}+D^{2}}$. For $D<D_{c}$ where $D_{c}$ satisfies the equation $\sqrt{D_{c}^{2}+J_{+}^{2}}=\left|J_{z}\right|+\left|J_{-}\right|$, the ground state of the system is the maximally entangled Bell state $|00\rangle-|11\rangle$. If we increase $D$, the difference between energy levels $E_{1}$ and $E_{3}$ decreases, so that at $D=D_{c}$ the ground state becomes degenerate and entanglement vanishes. When $D>D_{c}$ the ground state $E_{3}$ becomes entangled again.

Due to the mixture of states by increasing temperature the entanglement decreases, so that, in the under critical region $D<D_{c}$ the concurrence is

$$
C_{12}=\max \left\{\frac{\sinh \frac{\left|J_{-}\right|}{k T}-\cosh \frac{\sqrt{J_{+}^{2}+D^{2}}}{k T} e^{-\left|J_{z}\right| / k T}}{\cosh \frac{\left|J_{-}\right|}{k T}+\cosh \frac{\sqrt{J_{+}^{2}+D^{2}}}{k T} e^{-\left|J_{z}\right| / k T}}, 0\right\},
$$

while in the over critical region $D>D_{c}$ it is

$$
C_{12}=\max \left\{\frac{\sinh \frac{\sqrt{J_{+}^{2}+D^{2}}}{k T}-e^{\left|J_{z}\right| / k T} \cosh \frac{\left|J_{-}\right|}{k T}}{\cosh \frac{\sqrt{J_{+}^{2}+D^{2}}}{k T}+e^{\left|J_{z}\right| / k T} \cosh \frac{\left|J_{-}\right|}{k T}}, 0\right\} .
$$

For $D=D_{c}$, due to $\lambda_{1}=\lambda_{3}$, the entanglement vanishes for any temperature. The entanglement dependence on $T$ and $D$ is shown in Figs. 8 and 9. For $T=$ 0 , the figures show nonanalyticity at $D=D_{c}$ which signals a quantum phase transition. The entanglement behavior in the under and the over critical regions is qualitatively different. For the under critical case with fixed temperature the entanglement decreases with growing $D$, and the level of entanglement quickly decreases with temperature. From another side, for fixed temperature in the over critical region the entanglement increases, and the level of entanglement decreases with temperature quite slowly. In addition, if at $T=0$ we have only one critical point $D=D_{c}$ in which entanglement is zero, for $T>0$ entanglement vanishes at some interval which includes $D_{c}$ and this interval extends with growing temperature. This is a result of ground state mixture with higher states. However, by increasing $D$ we can always lower the level of our ground state to decrease this mixture and increase entanglement. 

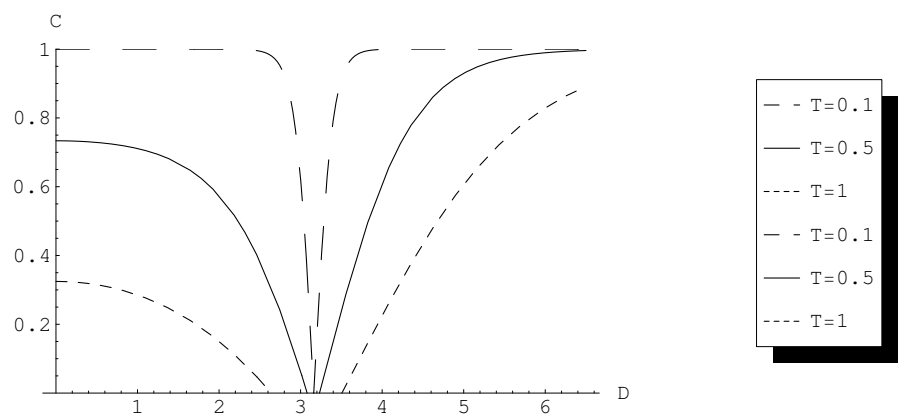

Fig. 8. Concurrence in ferromagnetic $X Y Z$ model versus coupling $D$ at temperature $T=0.1$, $0.5,1$.

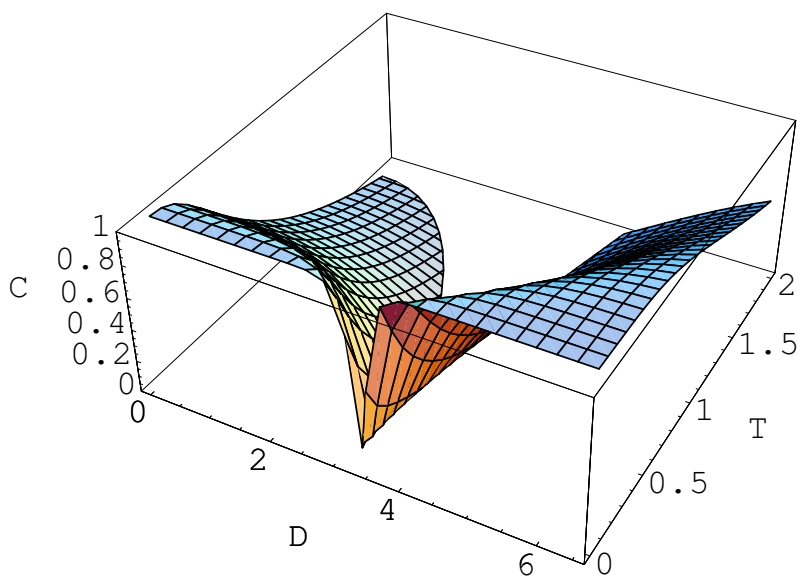

Fig. 9. 3D plot of concurrence in ferromagnetic $X Y Z$ model versus coupling $D$ and temperature $T$.

\section{Conclusion}

In conclusion, we like to stress several implications for future studies. As was shown by Alcaraz et al. ${ }^{35}$ the $X X Z$ quantum Heisenberg chain with the DM interaction is equivalent to the pure $X X Z$ Hamiltonian with modified boundary conditions and the anisotropy parameter, so that with these boundary conditions the model is still solvable by the Bethe Ansatz. Taking into account our results, it shows possibility to control entanglement in $X X Z$ model by varying boundary conditions.

Recently, it was found that the DM interaction can excite the entanglement and teleportation fidelity by using two independent Heisenberg $X X X$ chains. ${ }^{36}$ Moreover, studying the effect of a phase shift on amount transferable two-spin entanglement, ${ }^{37}$ it was shown that maximum attainable entanglement is enhanced by the DM interaction. Very recently, geometric computations for a spin chain model with the DM interaction has been discussed in Ref. 42. Finally it was found that the DM interaction is present in a number of quasi-one-dimensional magnets and is 
dominating for the compound $\mathrm{RbCoCl}_{3} \cdot 2 \mathrm{H}_{2} \mathrm{O}$. These indicate that DM interaction could be significant in designing the spin-based realistic quantum computers. ${ }^{38}$ The above mentioned results suggest to study the most general $X Y Z$ Heisenberg model with DM interaction as a quantum channel for quantum teleportation. These questions are now under investigation.

Finally, it is an interesting but analytically hard problem to study thermal entanglement in the multi-qubit spin models. Several partial results in this direction have been obtained recently by evaluating the pairwise entanglement of the two-site density matrix after tracing out all other spins in the chain: pairwise entanglement of three-qubit Heisenberg chain, ${ }^{29,43}$ pairwise entanglement of $N$ spin Heisenberg chain. Typical questions are to what extent nearest neighbors can be entangled with each other, or, how large the nearest-neighbor concurrence can be. ${ }^{44}$ In the Heisenberg model case, comparison of the nearest pairwise entanglement and the nonnearest one versus $T$ and $B$ has been investigated numerically. ${ }^{25}$ For the Ising model in the multi-qubit case, ${ }^{16}$ numerical results indicate that the behavior for even $N$ rings is quite similar to that of the two-qubit case. From another side, for nonneighboring qubits they observed that the even $N$ case has lower entanglement on average than the odd $N$ case. As the number $N$ of qubits in the chain is increasing, the difference between even and odd $N$ chains should disappear. Using the Jordan-Wigner transformation, the problem of $N$ qubits can be formulated in terms of one-dimensional spinless fermions. In the simplest case of $X Y$ model with transverse field the pairwise entanglement has been studied in Ref. 23. Some preliminary results suggest interesting directions to study: entanglement between a pair of particles is determined for the case where the pair is extracted from a symmetric state of $N$ two-level systems, ${ }^{45}$ the antiferromagnetic ring with even number of spins maximizes entanglement within a limited set of states, but not absolutely. ${ }^{44}$

\section{Acknowledgments}

One of the authors (Z.N.G.) would like to thank Dr. Koji Maruyama, for his helpful remarks. This work was supported partially by Izmir Institute of Technology, Turkey. The authors would like to thank the referee for useful remarks.

\section{References}

1. E. Schrödinger, Proc. Camb. Phil. Soc. 31, 555 (1935).

2. A. Einstein, B. Podolsky and N. Rosen, Phys. Rev. 47, 777 (1935).

3. J. S. Bell, Physics 1, 195 (1964).

4. C. H. Bennet and D. P. DiVincenzo, Nature 404, 247 (2000).

5. D. G. Angelakis, M. Christandl, A. Ekert, A. Kay and S. Kulik, Quantum Information Processing: From Theory to Experiment, NATO Science Series: Computer and Systems Sciences, Vol. 199, (IOS Press, 2006).

6. V. G. Baryakhtar, P. E. Wigen and N. A. Lesnik (eds.), Frontiers in Magnetism of Reduced Dimension Systems (Kluwer Academic Press, 1998).

7. E. H. Lieb and D. C. Mattis, Mathematical Physics in One Dimension: Exactly Solvable Models of Interacting Particles (Academic Press, 1966). 
8. R. J. Baxter, Exactly Solved Models in Statistical Mechanics (Academic Press, 1982).

9. S.-B. Zheng and G.-C. Guo, Phys. Rev. Lett. 85 (2000).

10. A. Imamoglu, D. D. Awschalom, G. Burkhard, D. P. DiVincenzo, D. Loss, M. Sherwin and A. Small, Phys. Rev. Lett. 83, 4204 (1999).

11. Y. Tong and R. Tao, Non-adiabatic arbitrary geometric phase gate in 2-qubit NMR model, quant-ph/0607041v3.

12. G. Yusa, K. Muraki, K. Takashina, K. Hashimito and Y. Hirayama, Nature 434, 1001 (2005).

13. I. L. Chuang, L. M. K. Vandersypen, X. Zhou, D. W. Leung and S. Lloyd, Nature 393, 143 (1998).

14. L. M. K. Vandersypen, M. Steffen, G. Breyta, C. S. Yannoni, M. H. Sherwood and I. L. Chuang, Nature 414, 883 (2001).

15. W. K. Wooters, Phys. Rev. Lett. 80 (1998).

16. D. Gunlycke, V. M. Kendon, V. Vedral and S. Bose, Phys. Rev. A 64, 0432302 (2001).

17. A. F. Terzis and E. Paspalakis, Phys. Lett. A 333 (2004).

18. A. M. Childs, D. W. Leung, F. Verstraete and G. Vidal, Quant. Inf. Comput. 3, 97 (2003).

19. X. Wang, Phys. Rev. A 66 (2002).

20. X. Wang, Phys. Rev. A 64 (2001).

21. G. L. Kamta and A. F. Starace, Phys. Rev. Lett. 88 (2002).

22. X. Xi and W. M. Liu, Chin. Phys. Soc. 16 (2007).

23. S. D. Hamieh and M. I. Katsnelson, Phys. Rev. A 72, 032316 (2005).

24. Y. Sun, Y. Chen and H. Chen, Phys. Rev. A 68 (2003).

25. M. C. Arnesen, S. Bose and V. Vedral, Phys. Rev. Lett. 87 (2001).

26. X. Wang, Phys. Lett. A 281, 101 (2001).

27. X. Xi, W. Chen, S. Hao and R. Yue, Phys. Lett. A 300, 567 (2002).

28. G. Rigolin, Int. J. Quant. Inf. 2, 393 (2004).

29. L. Zhou, H. S. Song, Y. Q. Guo and C. Li, Phys. Rev. A 68, 024301 (2003).

30. I. Dzialoshinski, J. Phys. Chem. Solids 4, 241 (1958).

31. T. Moriya, Phys. Rev. Lett. 120, 91 (1960).

32. W. K. Wooters, Phys. Rev. Lett. 78 (1997).

33. S. Sachdev, Quantum Phase Transitions (Cambridge Unversity Press, 1999).

34. M. A. Nielsen, Ph.D. thesis, University of Mexico, 2000.

35. W. F. Wreszinski and F. C. Alcaraz, J. Stat. Phys. 58, 45 (1990).

36. G.-F. Zhang, Thermal entanglement and teleportation in a two-qubit Heisenberg chain with DM anisotropic antisymmetric interaction, quant-ph/0703019v1.

37. K. Maruyama, T. Iitaka and F. Nori, Phys. Rev. A 75, 012325 (2007).

38. K. V. Kavokin, Phys. Rev. B 64, 075305 (2001).

39. J. N. McElearney and S. Merchant, Phys. Rev. B 18, 3612 (1999).

40. D. N. Aristov and S. V. Maleyev, Phys. Rev. B 62, R751 (2000).

41. A. S. T. Pires and M. E. Goueva, J. Magn. Mater. 212, 251 (2000).

42. J. A. Jones, V. V. Vedral, A. Ekert and G. Castagnoli, Nature 403, 869 (2000).

43. X. Wang, H. Fu and A. I. Solomon, J. Phys. A: Math. Gen. 34, 11307 (2001).

44. K. M. O'Connor and W. K. Wootters, Phys. Rev. A 63, 052302 (2001).

45. X. G. Wang and K. Molmer, Eur. Phys. J. D 18, 385 (2002). 
Copyright of International Journal of Modern Physics B: Condensed Matter Physics; Statistical Physics; Applied Physics is the property of World Scientific Publishing Company and its content may not be copied or emailed to multiple sites or posted to a listserv without the copyright holder's express written permission. However, users may print, download, or email articles for individual use. 